\title{
Evaluación de las competencias clínicas en una residencia de pediatría con el Mini-CEX (Mini-Clinical Evaluation Exercise)
}

\author{
Assessment of clinical competence in a pediatric residency with \\ the Mini-Clinical Evaluation Exercise (Mini-CEX)
}

\section{Dr. Gabriel Martín Fernández Gálvez ${ }^{a}$}

\section{RESUMEN}

Objetivo. Evaluar las competencias clínicas de los residentes de pediatría con la implementación del Mini-CEX, determinando su validez, confiabilidad, factibilidad y la satisfacción de docentes y de residentes.

Métodos. Participaron 14 docentes y 8 residentes. Se utilizó el Mini-CEX, método basado en la observación directa del desempeño del residente durante su práctica diaria, por parte de un docente.

Resultados. Se realizaron 181 observaciones, media de 12,92 observaciones por cada docente. Cada docente evaluó a 5,78 residentes. Hubo una media de 22,6 evaluaciones por residente. Las observaciones se realizaron en consultorios externos 38,7\%, internación pediátrica 19,3\%, neonatología $17,1 \%$, sala de recepción del recién nacido $14,4 \%$ y en internación conjunta $10,5 \%$. Los puntajes promedios fueron: profesionalismo 7,15; entrevista 6,64; examen clínico 6,67; criterio clínico 6,70; asesoramiento 6,79 y organización 6,73 . Los puntajes de competencia global variaron de acuerdo a los años de experiencia. Primer año 6,57; segundo 6,87 y tercero 7,$3 ; p=0,004$. El puntaje de satisfacción de los docentes fue 7,89 y de los residentes 7,74. El tiempo de duración de las observaciones fue de 28,35 minutos. El coeficiente alfa de Cronbach fue de 0,97 lo que indica elevada confiabilidad del método de evaluación. El ANOVA de puntajes de competencia global de todos los docentes mostró diferencias estadísticamente significativas, $\mathrm{p}<0,0001$.

Conclusiones. La implementación del Mini-CEX fue factible, bien aceptada por residentes y docentes, permitió valorar los diferentes niveles de desempeño de los residentes.

Palabras clave:educación médica, competencia, eva-

a. Residencia de

Pediatría, Docencia e Investigación. Policlínico Neuquén.

Correspondencia:

Gabriel Martín

Fernández Gálvez: fgalvez30@gmail.com

Conflicto de intereses: Ninguno que declarar.

Recibido: 17-5-2011

Aceptado: 28-6-2011 luación, ejercicio de examen clínico reducido (MiniCEX), residencia.

\section{SUMMARY}

Purpose. Assess the clinical competence of pediatric residents with the implementation of Mini-Clinical Evaluation Exercise (Mini-CEX), determining its validity, reliability, feasibility and satisfaction of examiners and residents. Methods. 14 examiners and 8 residents of pediatrics took part. The Mini-CEX, a method based on direct observation of residents during their daily training, was used. A nine-point rating scale was used in order to evaluate their skills regarding medical interviewing, physical examination, professionalism, clinical judgment, counselling, organization, overall competence and satisfaction with the method.

Results. 181 observations were made, an average of 12.92 observations per examiner (range-2-39). Each examiner assessed 5.78 residents, (range $2-8$ ). There was an average of 22.6 assessments per resident, range (18-30). The observations took place in outpatient clinic $38.7 \%$, pediatric inpatient unit $19.3 \%$, neonatal intensive care unit $17.1 \%$, neonatal reception unit $14.4 \%$ and rooming-in $10.5 \%$. The mean scores were: professionalism 7.15; interviewing 6.64; physical examination 6.67; clinical judgment 6.70 ; counselling 6.79 and organization 6.73 . The overall competence score varied according to experience levels. Mean scores were: first-year residents 6.57; second-year residents 6.87 and third-year residents $7.3 ; \mathrm{p}=0.004$. The score related to examiners's satisfaction was 7.89 and that of the residents was 7.74 . The duration of the observation period was 28.35 minutes. Cronbach alfa coefficient was 0.97 showing the high reliability of the assessment method. The ANOVA score for overall competence of all examiners showed statistically significant differences, $p<0.0001$ in relation to stricter or more lenient judgment to evaluate skills.

Conclusions. The implementation of the MiniCEX in the Pediatrics Residency was feasible and positively accepted by residents and examiners. It allowed the assessment of different levels of performance among residents according to their experience, in every clinical setting of a pediatrician's practice. The variability criteria among examiners and the lack of constructive criticism are matters to be dealt with in future investigations. Key words: medical education, clinical competence, assessment, mini clinical evaluation exercise (Mini CEX), residency.

\section{INTRODUCCIÓN}

La competencia puede definirse como la capacidad para utilizar el buen juicio, los conocimientos, habilidades y actitudes para solucionar problemas complejos que se presentan en distintos contextos en el campo de la actividad profesional. ${ }^{1}$

La competencia es contextual, refleja la relación entre las capacidades 
de una persona y las tareas que se requieren para el desempeño en una situación particular en el mundo real. La evaluación de las competencias es un desafío para los educadores médicos y muchos de los instrumentos que se utilizan para evaluarlas no permiten más que una visión parcial de las mismas. En la evaluación de la competencia profesional se debe procurar la utilización de instrumentos debidamente validados, que exploren no solo el conocimiento sino también las actitudes, destrezas y habilidades en escenarios apropiados. ${ }^{2}$ Las distintas áreas de competencias deberían evaluarse de manera integrada, coherente y longitudinal con el uso de múltiples métodos, proporcionando de manera frecuente feedback o devolución constructiva. ${ }^{3}$

George Miller (1990), desarrolló un modelo de competencia profesional representado por una pirámide compuesta de varios niveles. En la base se sitúan los conocimientos (el saber) sobre los que se apoya la competencia (el saber cómo). En un nivel superior se encuentra el desempeño (mostrar cómo) y finalmente la acción en la práctica real (el hacer). ${ }^{4}$ El nivel superior de la pirámide de Miller, el ser capaz de "hacer" en un contexto real, puede ser evaluado con el Mini Clinical Evaluation Exercise (Mini-CEX), que se centra en las habilidades que los residentes demuestran en el encuentro con el paciente y en donde el docente utiliza un formulario breve y estructurado durante la observación directa o por video. El Mini-CEX tiene su origen en Estados Unidos en la década de 1990 y fue desarrollado inicialmente por el American Board of Internal Medicine. ${ }^{5,6}$

El Mini-CEX es un método de observación directa de la práctica profesional con evaluación estructurada mediante un formulario y posterior devolución al residente. El tiempo promedio aconsejado es de 30 minutos, incluyendo la observación y la devolución. Los estudios de Holmboe et al., Norcini et al., y Alves de Lima et al., entre otros, confirman la validez, confiabilidad, factibilidad y satisfacción de este instrumento de evaluación. ${ }^{6-8}$

La formación de pediatras con las competencias necesarias para un ejercicio profesional que brinde calidad en la atención de la salud es uno de los objetivos de los programas de Residencias. Es necesario evaluar las competencias con métodos más objetivos, estructurados y sistemáticos. Nuestra hipótesis fue que el Mini-CEX es un instrumento válido y confiable para evaluar las competencias clínicas de los médicos residentes de pediatría. Los objetivos fueron: evaluar las competencias clínicas de los residentes de pediatría con la implementación del Mini-CEX, determinar su validez, confiabilidad y factibilidad. Evaluar la satisfacción de los docentes y de los residentes con el Mini-CEX.

\section{Procedimientos, participantes y métodos}

El diseño del estudio fue analítico cuantitativo y descriptivo. El Mini-CEX se implementó en la Residencia de Pediatría del Policlínico Neuquén, de la ciudad de Neuquén. La Residencia está acreditada por la Universidad Nacional del Comahue y por la Sociedad Argentina de Pediatría. Participaron del estudio todos los residentes de pediatría, dos de tercer año, dos de segundo año y cuatro de primer año.

Las observaciones del Mini-CEX se realizaron durante el segundo semestre de 2010, abarcando situaciones clínicas en consultorios externos, sala de internación pediátrica, Unidad de Cuidados Intensivos Neonatales (UCIN), sala de recepción del recién nacido y habitaciones de internación conjunta madre-recién nacido. El grupo de evaluadores estuvo compuesto por diez docentes de la residencia y cuatro docentes de la Facultad de Medicina de la Universidad Nacional del Comahue.

Las evaluaciones se realizaron durante la práctica profesional habitual de los residentes, en los distintos ámbitos de atención donde les correspondía actuar. Los docentes observaron y evaluaron la actuación del residente y anotaron en las fichas estandarizadas (Anexo 1) sus apreciaciones, considerando el grado de complejidad de la situación clínica, de acuerdo a su criterio en: bajo, moderado o alto. Se consignó si el enfoque de la observación estuvo destinado a la anamnesis, el diagnóstico, el tratamiento o el asesoramiento del paciente y la familia, o cualquier combinación de éstos.

Luego de la evaluación, se proporcionó una devolución constructiva al residente destacando las áreas en que se desempeñó correctamente y las que debe mejorar. El residente y el evaluador indicaron su grado de satisfacción con el Mini-CEX con un puntaje de 1 a 9 .

Las competencias evaluadas con el Mini-CEX fueron las siguientes: habilidades de entrevista clínica, habilidades de exploración física, profesionalismo, juicio clínico, habilidades comunicativas, organización/eficiencia y valoración global de la competencia. A cada competencia se otorgó un puntaje, de 1 a 3 se consideró insatisfactorio, 4 a 6, satisfactorio y de 7 a 9 sobresaliente. Dependiendo de la situación clínica propia de cada encuentro de evaluación, algunas de estas habilidades pudieron no ser observadas. También se 
consignó el tiempo de duración de la observación y de la devolución.

Para uniformar los criterios de observación y evaluación de las competencias, los docentes se guiaron por los descriptores definidos en el modelo del American Board of Internal Medicine. ${ }^{9}$ Antes de comenzar las observaciones se realizaron reuniones con los docentes para capacitarlos en el funcionamiento del Mini-CEX y en los mecanismos para la provisión de devolución inmediata. También hubo reuniones con los residentes sobre los objetivos de la evaluación y que se evaluarían sus competencias con fines formativos.

\section{Análisis estadístico}

La unidad de análisis fue cada encuentro de evaluación. Se realizó el análisis descriptivo de los encuentros, determinando la complejidad del caso, el foco de interés de la evaluación, el ámbito del encuentro, el sexo y edad del paciente. Se midió el puntaje obtenido por los residentes de cada año en las competencias evaluadas y el puntaje global alcanzado. Para el análisis descriptivo se determinaron las medias y desvíos estándares. La validez se evaluó por la capacidad del Mini-CEX de discriminar entre los niveles preexistentes de habilidades y competencias. Se aplicó la prueba no paramétrica de Kruskal Wallis para determinar las diferencias de habilidades entre los grupos de residentes, un valor de $\mathrm{p}<0,05$ se consideró estadísticamente significativo.

Se realizó un ANOVA de un factor para analizar la variabilidad de los puntajes entre los docentes.

Para determinar la consistencia interna del instrumento y su confiabilidad se calculó el coeficiente alfa de Cronbach para cada ítem de la escala considerando todas las evaluaciones de los docentes. La factibilidad se definió de acuerdo con el American Board of Internal Medicine, para las guías de implementación del Mini-CEX como un promedio de 4 evaluaciones por residente.

Para el análisis se utilizaron los paquetes estadísticos Infostat, ${ }^{10}$ STATA 10.0 y EpiInfo 2002.

\section{RESULTADOS}

En total se realizaron 181 observaciones con el Mini-CEX, participaron 14 docentes y 8 residentes. Hubo una media de 12,92 observaciones por cada docente, con un mínimo de 2 y un máximo de 39 observaciones. Los docentes evaluaron a 5,78 residentes cada uno en promedio, rango de 2 a 8 . Seis de los docentes realizaron observaciones a cada uno de los 8 residentes.
Del total de observaciones ( $n=181)$, el 50,8\% correspondió a residentes de primer año (R1), $28,7 \%$ a residentes de segundo año (R2), y 20,5\% a residentes de tercer año (R3). Hubo una media de 22,6 evaluaciones por residente (rango: 18-30).

Cada residente fue evaluado por varios docentes (mínimo 8 y máximo 11).

En la Tabla 1 se observa la cantidad de observaciones realizadas en cada uno de los escenarios clínicos.

La complejidad de la consulta o del acto médico fue considerada: baja en 50,3\%, moderada en $40,9 \%$ y alta en $8,8 \%$ de las observaciones. Las consultas fueron de "primera vez" en 75,1\% y de "seguimiento" en $24,9 \%$ de los casos.

El énfasis de la observación recayó sobre la anamnesis en $80 \%$ de las evaluaciones, el diagnóstico en $75 \%$, la terapéutica en $66 \%$ y el asesoramiento en el $77 \%$. La edad de los pacientes atendidos abarcó todo el espectro de edades pediátricas. Los pacientes fueron $53,6 \%$ de sexo masculino y $46,4 \%$, femenino.

Entre las competencias evaluadas, el puntaje más alto fue para profesionalismo: 7,15 $( \pm 1,17)$ y los más bajos para entrevista o anamnesis: 6,64 $( \pm 1,31)$ y para examen clínico: $6,67( \pm 1,37)$. Los otros puntajes fueron: criterio clínico: $6,79( \pm 1,26)$; asesoramiento: 6,79 ( $\pm 1,34)$; organización: 6,73 ( \pm $1,40)$ y competencia global: $6,80( \pm 1,24)$. El tiempo de duración total de las observaciones fue de $28,35$ minutos en promedio ( \pm 17$)$, incluyendo la devolución proporcionada al residente. La satisfacción de los docentes tuvo un puntaje promedio de $7,89( \pm 0,67)$ y la de los residentes $7,74( \pm 0,74)$. La satisfacción de los residentes se correlacionó con el puntaje de competencia global obtenido y con el docente que realizaba la evaluación. Los puntajes promedio de satisfacción de los residentes para las evaluaciones de cada docente osciló entre 6,64 a 8,67, $p<0,0001$.

Se realizó el análisis comparativo, según el

TABLA 1. Frecuencia de evaluaciones en cada escenario clínico

\begin{tabular}{lcc}
\hline Ámbito de la observación & Frecuencia & Porcentaje \\
\hline Consultorios externos & 70 & 38,7 \\
Internación pediátrica & 35 & 19,3 \\
UCIN (Unidad Cuidados & & \\
Intensivos Neonatales) & 31 & 17,1 \\
Recepción del recién nacido & 26 & 14,4 \\
Internación conjunta & 19 & 10,5 \\
Total & 181 & 100,0 \\
\hline
\end{tabular}


año de residencia, de los puntajes obtenidos en cada una de las competencias evaluadas (Tabla 2). Se observó una diferencia estadísticamente significativa entre los puntajes de competencia global entre los diferentes años de la residencia, $\mathrm{p}=0,004$.

El puntaje obtenido por los residentes en su competencia global varió de acuerdo al escenario clínico donde se desarrolló la observación. El puntaje más elevado fue en neonatología, con un puntaje medio de 7,45 $( \pm 0,77)$, el más bajo en sala de internación pediátrica con $6,29( \pm 1,33), \mathrm{p}=0,002$. El tiempo de duración del Mini-CEX, la satisfacción de los docentes y la de los residentes también tuvieron una variación estadísticamente significativa de acuerdo al escenario clínico (Tabla 3).

Se calculó el alfa de Cronbach para cada competencia considerando todas las evaluaciones de los docentes. El coeficiente de correlación entre ítems varió entre 0,91 y 0,97 , lo que indica muy buena consistencia interna entre los ítems del instrumento. El coeficiente alfa de Cronbach fue de 0,97 , lo que indica elevada confiabilidad del método de evaluación.

Se realizó un ANOVA de un factor entre los puntajes de competencia global de cada uno de los docentes y se observó una diferencia estadísticamente significativa entre los mismos, $\mathrm{p}<0,0001$.

\section{DISCUSIÓN}

El Mini-CEX conjuga bastante bien los criterios descriptos por Van der Vleuten, ${ }^{11}$ para determinar la utilidad de un método de evaluación. Tiene validez ya que permite diferenciar entre diferentes niveles de experiencia entre los residentes. ${ }^{5-8}$

Tiene confiabilidad, ya que múltiples encuentros, con diferentes pacientes y situaciones clínicas y distintos observadores, permiten obtener conclusiones sobre la competencia clínica global. Diferentes autores afirman que son necesarios entre 10 y 15 encuentros para que la evaluación sea confiable y los resultados reproducibles. ${ }^{7,5,12,13}$ Tiene un gran impacto educativo dado por lo significativo de la devolución constructiva en el aprendizaje futuro del residente. ${ }^{14,15}$

Es aceptado favorablemente por docentes y residentes, de acuerdo a los niveles de satisfacción mostrados por diferentes estudios incluyendo el presente. ${ }^{5-8,16}$

La implementación del Mini-CEX durante este estudio, permitió evaluar a los residentes en todos los escenarios clínicos de la práctica diaria de un pediatra. Fue factible de implementar ya que se desarrolló en un solo programa formativo y se hicieron más de 22 observaciones por residente en promedio, muchas más que las informadas por otros autores que variaron entre 2 y 4 evaluaciones. $6,8,16$

TABLA 2. Puntajes obtenidos en cada año de residencia

\begin{tabular}{lcccccccccc}
\hline & \multicolumn{3}{c}{ Residentes de $\mathbf{1}^{\text {er }}$ año } & \multicolumn{3}{c}{ Residentes de $\mathbf{2}^{\text {do }}$ año } & \multicolumn{3}{c}{ Residentes de ${ }^{\text {er }}$ año } \\
\hline Área evaluada & $\mathbf{n}$ & Media & D.E. & $\mathbf{n}$ & Media & D.E. & $\mathbf{n}$ & Media & D.E. & Valor de $\mathbf{p}$ \\
\hline Entrevista & 83 & 6,45 & 1,37 & 9 & 6,86 & 1,19 & 31 & 6,81 & 1,30 & $\mathrm{p}=0,10$ \\
Examen clínico & 85 & 6,47 & 1,39 & 46 & 6,72 & 1,33 & 34 & 7,09 & 1,31 & $\mathrm{p}=0,05$ \\
Profesionalismo & 90 & 7,01 & 1,13 & 52 & 7,25 & 1,22 & 35 & 7,37 & 1,19 & $\mathrm{p}=0,14$ \\
Criterio clínico & 90 & 6,61 & 1,22 & 49 & 6,76 & 1,38 & 36 & 7,28 & 1,09 & $\mathrm{p}=0,01$ \\
Asesoramiento & 81 & 6,67 & 1,4 & 47 & 6,74 & 1,36 & 30 & 7,2 & 1,06 & $\mathrm{p}=0,20$ \\
Organización & 91 & 6,46 & 1,36 & 51 & 6,78 & 1,51 & 37 & 7,3 & 1,15 & $\mathrm{p}=0,002$ \\
Competencia global & 92 & 6,57 & 1,24 & 52 & 6,87 & 1,28 & 37 & 7,3 & 1,05 & $\mathrm{p}=0,004$ \\
\hline
\end{tabular}

TABLA 3. Relación entre las variables y el escenario clínico de la observación

\begin{tabular}{lccccccccccc}
\hline & \multicolumn{2}{c}{$\begin{array}{c}\text { Consultorios } \\
\text { externos }\end{array}$} & \multicolumn{2}{c}{$\begin{array}{c}\text { Internación } \\
\text { conjunta }\end{array}$} & \multicolumn{2}{c}{$\begin{array}{c}\text { Internación } \\
\text { pediatría }\end{array}$} & \multicolumn{2}{c}{$\begin{array}{c}\text { Recepción del } \\
\text { recién nacido }\end{array}$} & \multicolumn{2}{c}{ UCIN } & \\
\hline Variable & Media & D.E. & Media & D.E. & Media & D.E. & Media & D.E. & Media & D.E. & Valor de p \\
\hline Competencia global & 6,81 & 1,44 & 6,63 & 1,07 & 6,29 & 1,13 & 6,81 & 1,1 & 7,45 & 0,77 & $\mathrm{p}=0,002$ \\
Tiempo de observación (min) & 14,94 & 4,52 & 19,58 & 5,82 & 16,03 & 5,11 & 24,38 & 10 & 31,19 & 25,19 & $\mathrm{p}<0,0001$ \\
Tiempo de devolución (min) & 8,01 & 3,84 & 8,53 & 3,27 & 9,23 & 3,88 & 8,77 & 5,1 & 8,94 & 3,02 & $\mathrm{p}=0,32$ \\
Satisfacción del docente & 8,09 & 0,68 & 7,79 & 0,42 & 7,54 & 0,74 & 7,85 & 0,8 & 7,94 & 0,44 & $\mathrm{p}=0,001$ \\
Satisfacción del residente & 7,93 & 0,71 & 7,74 & 0,56 & 7,34 & 0,76 & 7,73 & 0,8 & 7,77 & 0,72 & $\mathrm{p}=0,002$ \\
\hline
\end{tabular}


El tiempo de duración de los encuentros fue de 28 minutos, aproximadamente 20 minutos de observación y 8 de devolución, variando de acuerdo a las características propias de cada situación clínica. Este tiempo coincide con lo demostrado por otros estudios donde la observación fue entre 20 y 26 minutos y la devolución, 7 minutos. ${ }^{6,17} \mathrm{Sin}$ embargo, se debería poner más énfasis en el tiempo dedicado a la devolución dada la importancia que tiene. En este sentido el tiempo de 8 minutos parece insuficiente, comparado con los 15 minutos del estudio de Eriksen et al., o los 17 minutos reportados por Alves de Lima, et al. 18,8

El puntaje de competencia global medio para los residentes de primer año fue 6,57, para los de segundo año 6,87 y para los de tercer año 7,3; observándose una diferencia estadísticamente significativa, de modo que el Mini-CEX permitió discriminar entre los diferentes niveles de competencia global entre los residentes de los diferentes años, lo que coincide con otros estudios y reafirma la validez del instrumento. ${ }^{5-8}$ Wiles et al., evaluaron a médicos en formación en neurología, en la Universidad de Cardiff en el Reino Unido, encontrando que el puntaje de competencia global y de habilidades de comunicación aumentaron con significancia estadística con cada año de entrenamiento. ${ }^{19}$

En el presente estudio el puntaje obtenido por los residentes en su competencia global varió de acuerdo al escenario clínico donde se desarrolló la observación. Algunos autores encontraron mayores puntajes en pacientes internados que en consultorios externos; esto puede deberse a que una mayor complejidad de la consulta se correlaciona con puntajes más altos. ${ }^{6,17}$ Otros observaron que el puntaje de competencia global fue similar en todos los escenarios clínicos. ${ }^{8}$

El coeficiente alfa de Cronbach fue de 0,97 y el coeficiente de correlación entre ítems varió entre 0,91 y 0,97 . Este resultado es similar al encontrado por Cook et al., quienes reportaron un coeficiente alfa de Cronbach mayor a 0,86 para los seis ítems de la escala, y a los resultados de Megale, et al., para quienes el alfa de Cronbach varió entre 0,87 y 0,96 . Estos resultados indican alta confiabilidad y consistencia interna entre los ítems del instrumento. ${ }^{20,21}$

$\mathrm{Al}$ realizar el test ANOVA de un factor a los puntajes de competencia global de todos los docentes se observan diferencias estadísticamente significativas, $p<0,0001$, en relación a criterios más estrictos o más indulgentes para evaluar las competencias.
Si bien las diferencias de criterios de los docentes pueden afectar la confiabilidad del método de evaluación, está demostrado que, al aumentar el número de docentes que realizan los encuentros, se minimiza este problema, ya que esto disminuye la variabilidad interobservador. ${ }^{17,22-24}$ Se puede aumentar la objetividad de la evaluación con la formación y entrenamiento de los docentes, para mejorar las observaciones y adoptar criterios uniformes durante las mismas. ${ }^{15}$

La observación directa es uno de los instrumentos más útiles para evaluar el desarrollo de las habilidades clínicas de los residentes y el rol de los educadores es fundamental, por lo que la formación de los docentes y la provisión de devolución son críticas para el éxito de un programa de observación directa. ${ }^{25,26}$

Una preocupación constante de los educadores médicos debe ser el impacto educativo de los métodos de evaluación y de allí la importancia que reviste la devolución. Ésta mejora el aprendizaje, favorece la motivación y las habilidades de autorregulación, es decir la capacidad para reconocer debilidades y fortalezas y así identificar áreas que requieran mejor desempeño. Durante la devolución constructiva posterior al Mini-CEX, el docente puede evaluar la capacidad de autocrítica y de reflexión del residente, y guiarlo para que la desarrolle. De esta manera se estimula la capacidad de aprender a aprender, que es quizás, la mejor enseñanza que se le puede brindar al residente, ya que esta capacidad le acompañará durante toda su vida. ${ }^{27-29}$

Limitaciones del presente estudio: el número de residentes que participó fue bajo y los resultados tienen relación directa con factores contextuales propios del programa de formación donde fue implementado el Mini-CEX, por eso es posible que estos resultados no puedan ser extrapolados a otras residencias. La presente experiencia debería reproducirse en centros con mayor número de residentes para que los resultados permitan hacer generalizaciones.

\section{CONCLUSIONES}

La implementación del Mini-CEX en la Residencia de Pediatría fue factible, bien aceptada por los residentes y los docentes, permitió valorar los diferentes niveles de desempeño de los residentes según su experiencia, con adecuada validez y confiabilidad.

\section{Agradecimientos}

A los docentes que participaron en las evalua- 
ciones, al Dr. Raúl A. Borracci y al Dr. Alberto Alves de Lima por su colaboración en el análisis estadístico.

\section{BIBLIOGRAFÍA}

1. Brailovsky C. Evaluación de las Competencias. Aportes para un cambio curricular en Argentina. Facultad de Medicina-UBA. OPS. Buenos Aires, 2001. Disponible en: www. fmv-uba.org.ar/proaps/cambio1.htm.

2. Castro C, Ferrero F. Evaluación de la competencia profesional. Arch Argent Pediatr 2002;100(6).

3. Epstein RM. Assessment in medical education. $\mathrm{N}$ Engl J Med 2007;356:387-96.

4. Miller GE. The assessment of clinical skills/competence/ performance. Acad Med (Suppl) 1990;65:S63-S7.

5. Norcini J, Blank LL, Arnold GK, Kimball HR. The MiniCEX (Clinical Evaluation Exercise): a preliminary investigation. Ann Intern Med 1995;123:795-9.

6. Norcini J, Blank LL, Dufy FD, Fotna GS. The Mini-CEX: a method for assessing clinical skills. Ann Intern Med 2003;138:476-81.

7. Holmboe E, Huot S, Chung J, Norcini J, Hawkins R. Construct validity of the Mini-Clinical Evaluation Exercise (MiniCEX). Acad Med 2003;78:826-30.

8. Alves de Lima A, Barrero J, Baratta C, Costa S, Castillo, et al. Validity, reliability, feasibility and satisfaction of the Mini-Clinical Evaluation Exercise (Mini-CEX) for cardiology residency training. Med Teach 2007;29(8):785-90.

9. Fornells-Vallés JM. El ABC del Mini-CEX. Educ Med 2009;12(2):83-9.

10. Di Rienzo JA, Casanoves F, Balzarini MG, González L, et al. InfoStat versión 2010. Grupo InfoStat, FCA, Universidad Nacional de Córdoba, Argentina.

11. Van Der Vleuten C. The assessment of professional competence: developments, research and practical implications. Adv Health Sci Educ 1996;1:41-67.

12. Nair BR, Alexander HG, McGrath BP, Parvathy MS, et al. The mini clinical evaluation exercise (mini-CEX) for assessing clinical performance of international medical graduates. Med J Aust 2008;189:159-61.

13. Hill F, Kendall K, Galbraith K, Crossley J. Implementing the undergraduate mini-CEX: a tailored approach at Southampton University. Med Educ 2009;43(4):326-34.

14. Alves de Lima A, Henquin R, Thierer J. A qualitative study of the impact on learning of the mini-clinical evaluation exercise in postgraduate training. Med Teach 2005;27 (1):46-52.

15. Holmboe E, Yepes M, Williams F, Huot S. Feedback and the Mini Clinical Evaluation Exercise. J Gen Intern Med 2004;19:558-61.
16. Grosman A, Urman G, Urman J, Folgueral S, Alves de Lima A. Evaluación por competencias en pediatría: respondiendo al desafío. $58^{\circ}$ Congreso de la Asociación Española de Pediatría.4-6 de junio de 2009 [Acceso: 10 oct de 2010]. Disponible en: http:/ / www.aeped.es/eventos/58congreso-aep.

17. Wilkinson J, Crossley JG, Wragg A, Mills P, et al. Implementing workplace-based assessment across the medical specialties in the United Kingdom. Med Educ 2008;42:364-73.

18. Eriksen JG, Simonsen D, Bastholt L, Aspegren K, et al. Mini clinical evaluation exercise as evaluation tool of communicative and cooperative skills in the outpatient clinic. Ugeskr Laeger 2009;71(12):1003-6.

19. Wiles CM, Dawson K, Hughes TA, Llewelyn JG, et al. Clinical skills evaluation of trainees in a neurology department. Clin Med 2007;7(4):365-9.

20. CookDA. Twelve tips for evaluating educational programs. Med Teach 2010;32:296-301.

21. Megale L, Dias Gontijo E, César Motta JA. Avaliação de competência clínica em estudantes de medicina pelo Miniexercício Clínico Avaliativo (Miniex). Rev Bras Educ Med 2009;166,33(2):166-75.

22. Wass V, Van der Vleuten C, Shatzer J, Jones R. Assessment of clinical competence. Lancet 2001;357:945-9.

23. Margolis MJ, Clauser BE, Cuddy M, Ciccone A, et al. Use of the Mini-Clinical Evaluation Exercise to rate examinee performance on a multiple-station clinical skills examination: a validity study. Acad Med 2006;81(10 Suppl): S56S60.

24. Boulet JR, Mckinley D, Norcini JJ, Whelan GP. Assessing the comparability of standardized patient and physician evaluations of clinical skills. Adv Health Sci Educ Theory Pract 2002;7:85-97.

25. Hauer KE, Holmboe ES, Kogan JR. Twelve tips for implementing tools for direct observation of medical trainees \& clinical skills during patient encounters. Med Teach 2010;9:27.

26. Fromme HB, Karani R, Downing SM. Direct observation in medical education: a review of the literature and evidence for validity. Mt Sinai J Med 2009;76(4):365-71.

27. Alves de Lima A. Devolución constructiva una estrategia para mejorar el aprendizaje. Medicina (Bs. Aires) 2008; 68:88-92.

28. Carr S. The Foundation Programme assessment tools: An opportunity to enhance feedback to trainees? Postgrad Med J 2006;82:576-9.

29. Norcini JJ, Burch V. Workplace-based assessment as an educational tool: AMEE Guide No. 31. Med Teach 2007; 29(9):855-71. 
Anexo I

\section{EJERCICIO DE EXAMEN CLÍNICO REDUCIDO (EECR)}

EJERCICIO DE EXAMEN CUINICO REDUCIDO (EECR)

EVALUADOR:

RESIDENTE:

PROBLEMA DEL PACIENTE/DIAGNÓSTICO:

ÁMBITO

CONSULTORIOS

EXTERNOS

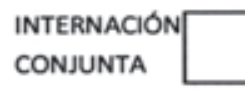

FECHA:

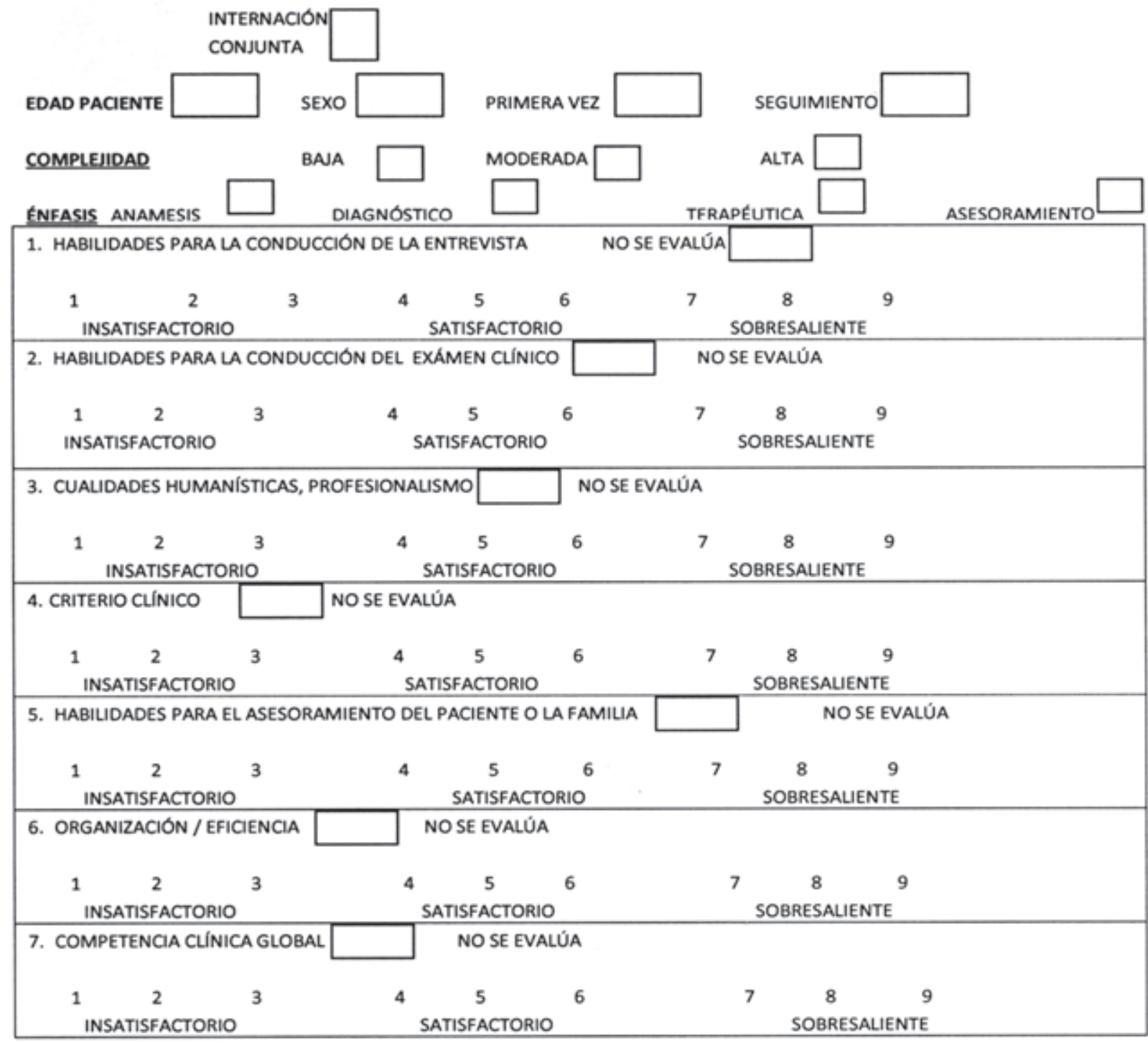

TIEMPO DE DESARROUO DEL EECR (minutos) SATISFACCIÓN DEL EXAMINADOR CON EL EECR $\begin{array}{lcccr}\text { BAIA } & 1 & 2 & 3 & 4 \\ \text { SATISFACCIÓN DEL RESIDENTE } & \text { CON EL EECR }\end{array}$ COMENTARIOS

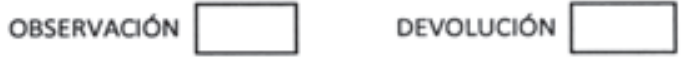

$\begin{array}{llllll}5 & 6 & 7 & 8 & 9 & \text { ALTA } \\ 5 & 6 & 7 & 8 & 9 & \text { ALTA }\end{array}$

